\title{
HARDWARE ROUGH SET PROCESSOR PARALLEL ARCHITECTURE IN FPGA FOR FINDING CORE IN BIG DATASETS
}

\author{
Maciej Kopczyński, Tomasz Grześ \\ Faculty of Computer Science, Bialystok University of Technology, \\ E-mail:t.grzes@pb.edu.pl
}

Submitted: 5th May 2020; Accepted: 5th November 2020

\begin{abstract}
This paper presents FPGA and softcore CPU based solution for large datasets parallel core calculation using rough set methods. Architectures shown in this paper have been tested on two real datasets running presented solutions inside FPGA unit. Tested datasets had 1000 to 10000000 objects. The same operations were performed in software implementation. Obtained results show the big acceleration in computation time using hardware supporting core generation in comparison to pure software implementation.

Keywords: rough sets, FPGA, core attributes, parallel architecture
\end{abstract}

\section{Introduction}

The biggest problem associated with creating optimal decision support systems is the time needed to process input data, with particular emphasis on large data sets, the so-called big data. Big data is a term referring to large, diverse and variable data sets, whose processing and analysis is difficult but at the same time valuable, because it can lead to the acquisition of new knowledge. In practical applications, the concept of a large data set is relative and means a situation when the set cannot be processed using simple and commonly available methods [17]. Depending on the purpose of the chosen method and the complexity of the algorithm, this may mean a calculated size in megabytes, gigabytes or terabytes.

One of the examples of such huge datasets are data read from sensors - like from production line. Every part of such line has tens of sensors providing data all the time, which creates huge stream of readings. Amount of obtained data creates huge datasets that has to be processed for e.g. estimating future failures and thus avoiding downtime.

There were many methods created for processing such datasets and focusing on knowledge extraction and minimizing amount of data that has to be processed. One of them are rough sets theory allowing to easily limit number of attributes (columns) in input datasets to these, that are provide the valuable information. In these methods two definitions exist - reduct and core. Reducts are sets of columns that provide useful information considering removing redundant information, while cores are sets of attributes that cannot be removed from initial set under any circumstances. In other words, the attributes existing in the core must also exist in all the reducts.

The rough sets theory developed in the eighties of the twentieth century by Prof. Z. Pawlak is an useful tool for data analysis. Therefore a lot of rough sets algorithms were implemented in scientific and commercial tools for data processing. But data processing efficiency problem is arising with 
the increase of the amount of data. Huge datasets are commonly referred to as Big Data.

Some of the solutions developed and oriented for data-type software implementations and algorithms with smaller computational complexity are shown below. An example of such an approach is research done in [1]. The authors presented an algorithm for feature extraction for a specific type of data with linear complexity. An additional software approach to dealing with big datasets involves parallel processing. One of the methods is related to the implementation of algorithms using MapReduce. The parallel technique for computing rough set approximations is presented in [28]. Algorithms for core and reduct calculation based on the distributed programming model of MapReduce can be found in [3]. Other types of approaches to fast processing data sets were shown in [4], where Binarized Neural Networks (BNN) were presented and are based on the optimization of computational complexity and memory demand. In [6], a framework for integrating hardware and software optimizations for sparse CNNs is shown. It is worth adding, that [7] presented an AlphaGo Policy Network using DCNN accelerator on FPGA.

Another solution for processing big datasets are hardware implementations of existing algorithms. This type of approach can be accomplished using FPGA units. Field Programmable Gate Arrays (FPGAs) are group of digital integrated circuits. Functionality of these units can be programmed by engineer at any time. This feature gives the possibility of evaluating any boolean function. That's why they can be used for supporting rough sets calculations, especially if algorithms of polynomial complexity and ability of processing parts of data in parallel are considered.

At the moment there are some hardware implementations of specific rough set methods. The idea of sample processor generating decision rules from decision tables was described in [21]. In [14] the authors presented the architecture of rough set processor based on cellular networks described in [16]. In [8] a concept of hardware device capable of minimizing the large logic functions created on the basis of discernibility matrix was developed. A more detailed summary of the existing ideas and hardware implementations of rough set methods can be found in [9] and in [27]. It should be noted, that [19] intro- duced efficient heuristics for computing from data table basic tools (like lower and upper approximations, positive regions, reducts) for rough set methods. Their heuristics are feasible for large data tables but are mainly focused on software solutions, while our proposition is focused on the hardware approach. In [18] implementation of the LEM2 algorithm on FPGA is presented.

Previous authors' research results focused on the subject of hardware implementations of rough sets methods can be found in previously published papers: the idea of the processor for rough sets methods [25], hardware-supported reduct calculation [10], the first approach to core generation using FPGA based solution [11], hardware unit for processing datasets consisting of millions of objects [12], a two-stage algorithm for finding reduct [5] as well as finding minimal reduct with two FPGA devices [2].

This paper is a new proposition for parallel hardware supported core calculation for big datasets and introduces modification of methods described in [11] and [13]. According to [11], the main focus was put on small datasets (up to 107 objects) and all operations were implemented as combinational logic, which gave huge acceleration in calculation speed, but was very limited to the amount of data, that could be processed. In [13], the target was to perform a sequential operation on core calculation based on the idea presented in [11], but this approach was limited to a single core.

The paper is organized as follows. In Section 2 some information about the notion of core and datasets used during research is provided. It includes also the pseudocode of the algorithms. Section 3 focuses on description of hardware solutions, while Section 4 is devoted to the experimental results.

\section{Introductory Information}

\subsection{The Notion of Core in the Rough Set Theory}

Let $D T=(U, A \cup\{d\})$ be a decision table, where $U$ is a set of objects, $A$ is a set of condition attributes and $d$ is a decision attribute. In decision table some of the condition attributes from $A$ may be superfluous (redundant in other words). 
This means that their removal cannot worsen the classification. The set $C \subseteq A$ of all indispensable condition attributes is called the core. None of its elements can be removed without affecting the classification power of all condition attributes. In order to compute the core we can use discernibility matrix $[D M(x, y)]_{x, y \in U}$, where $D M(x, y)=\{a \in A: a(x) \neq$ $a(y)$ and $d(x) \neq d(y)\}$.

The core is the set of all single element entries of the discernibility matrix, i.e. CORE = $\bigcup_{x, y \in U, \text { cardinality }(D M(x, y))=1} D M(x, y)$. A much more detailed description of the concept of the core can be found, for example, in the article [22] or in the book [24].

Algorithm presented in following subsections are based on available knowledge, but were substantially redesigned to provide possibility of their implementation in hardware units. Biggest effort was put on parallelism and identification of most computation-time consuming parts.

\subsection{Principle of Core Calculation}

One of the most common algorithm for core calculation using discernibility matrix is presented as pseudocode in this subsection. The biggest limitation of this algorithm using direct implementation, mainly for big datasets, is need for storing discernibility matrix $D M$ as two dimensional array with size of $|U| x|U|$. Hardware version of core calculation algorithm called CORE-PHIDM (described in Section 2.3) uses principles of this approach to core creation.

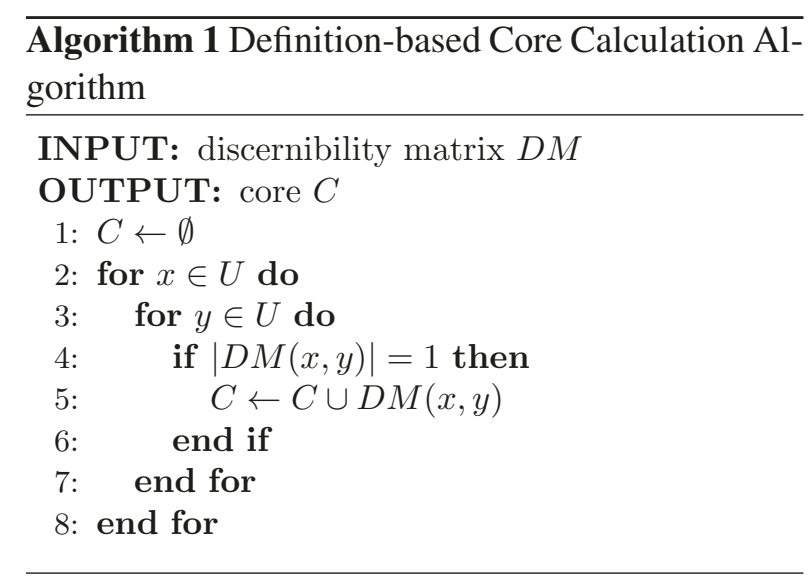

Input to the definition-based algorithm is discernibility matrix $D M$ and output is core $C$. Core is initialized as empty set in line 1. Two loops in lines
2 and 3 iterate over all objects (denoted as $U$ ) in discernibility matrix. Condition instruction in line 4 checks if matrix cell contains only one attribute. If so, then this attribute is added to the core $C$.

\subsection{Algorithm CORE-PHIDM for Hard- ware Supported Core Calculation With No Discernibility Matrix}

The main concept of CORE-PHIDM (CORE Parallel Hardware Indirect Discernibility Matrix) algorithm is based on a property of singleton, which is cell from discernibility matrix consisting of the only one attribute. This property tells that any singleton cannot be removed without affecting the classification power. As it was mentioned, biggest problem with direct use of discernibility matrix based algorithms when dealing with big datasets is amount of memory needed to store the matrix. Such solution cannot be run in hardware because of FPGA resources limitations.

This is the reason why we proposed the hardware implementable algorithm - CORE-PHIDM. Main idea is to divide the entire dataset into parts stored in multiple independent memory units providing information for hardware modules. These parts are subsequently processed by the units. For clarity of presentation, pseudocode of the algorithm shows the most simple configuration of COREPHIDM block: one instance of SUbCORE module with two memory blocks denoted as $R A M_{c m n}$ (common RAM connected to all modules) and $R A M_{1}$ (first $R A M_{n}$ for single and only subCORE module). Details related to hardware implementation and naming is described in Section 3. Pseudocode for the algorithm is given below: 


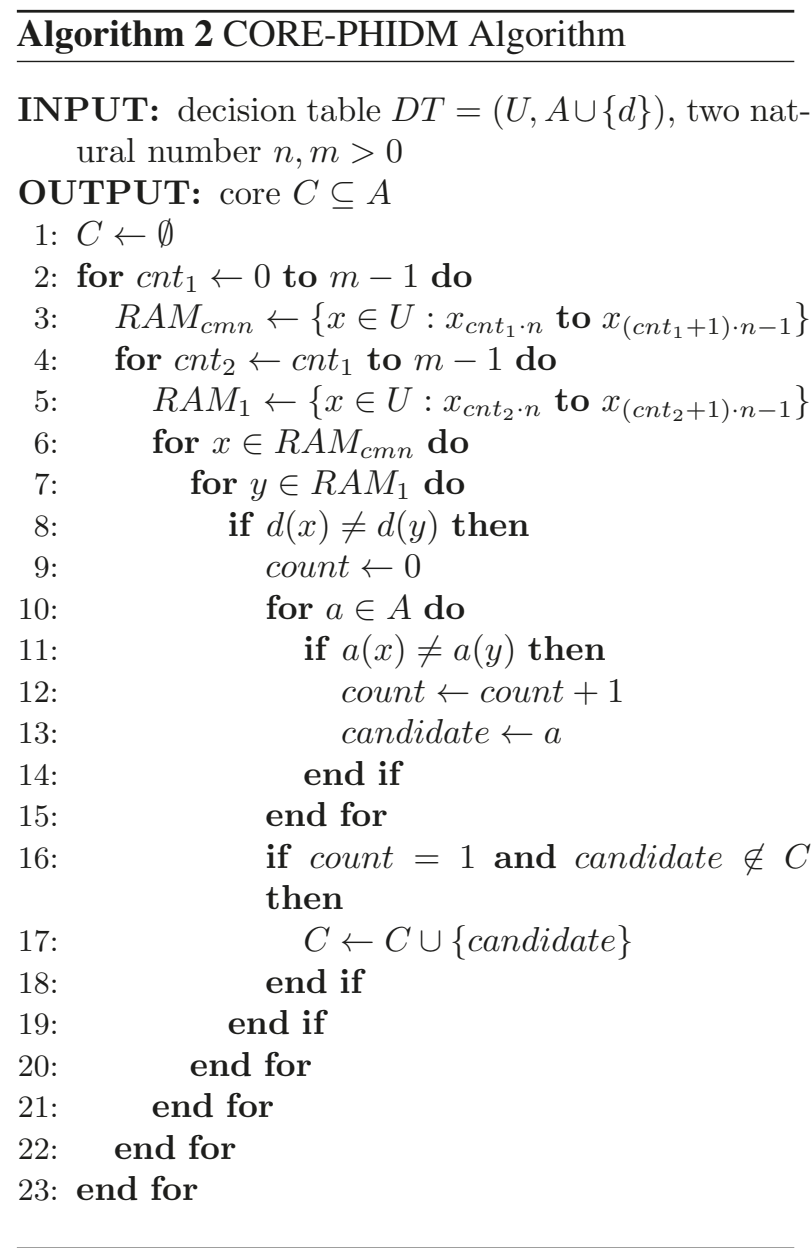

Input to the algorithm CORE-PHIDM is decision table $D T$, and output is core $C$. In the first step core $C$ is initialized as empty set. Two loops in lines 2 and 4 are responsible for choosing parts of input decision table. Decision table $D T$ is divided into $m$ parts, where each of them have the size of $n$ objects. Lines 3 and 5 are responsible for loading chosen parts of dataset into RAM memories implemented in FPGA. Two loops in lines 6 and 7 take subsequent objects from decision table parts for comparison. Line 8 performs the comparison between decision attribute's value of two objects $x$ and $y$. If these two objects belong to different decision classes, the rest of the algorithm is processed. count variable, responsible for storing the number of differences on condition attributes values between objects $x$ and $y$ is set to 0 in line 9. Loop in line 10 iterates over set of condition attributes $A$. Values of $a$ condition attribute is compared between objects $x$ and $y$ in line 11. In case of difference, the count variable is incremented and $a$ attribute is stored in candidate variable. When the attribute loop finishes, attribute in candidate variable is added to the core if count variable is equal to 1 and this attribute is not in core (lines 16 to 18 ).

It should be noted, that CORE-PHIDM module in complex configurations, where more than one subCORE block is used, loads more subsequent parts of $D T$ into the $R A M_{n}$ memories (line 5). Similarly, comparisons between decision attribute's values and condition attributes' values for objects take place between object selected from $R A M_{c m n}$ and objects selected from each $R A M_{n}$ block of existing SUbCORE modules (lines 8 to 19).

\subsection{Example of Core Calculation for Definition-based Algorithm}

Table 1 presents exemplary decision table consisting of 4 condition attributes and one decision attribute. Dataset describes decision related to concrete mixing on basis of thresholded and descriptive readings from 4 types of sensors: outlook, temperature value, humidity and wind strength.

Table 1. Exemplary dataset consisting of 4 condition and 1 decision attribute

\begin{tabular}{cccccc}
\hline ID & outlook & temp & humidity & windy & mixing \\
\hline 1 & sunnny & high & high & no & no \\
\hline 2 & sunnny & high & high & yes & no \\
\hline 3 & cloudy & high & high & no & yes \\
\hline 4 & sunnny & low & high & no & yes \\
\hline 5 & sunnny & high & normal & no & yes \\
\hline 6 & sunnny & high & normal & yes & no \\
\hline 7 & cloudy & high & normal & yes & yes \\
\hline 8 & sunnny & low & high & no & no \\
\hline 9 & sunnny & high & normal & no & yes \\
\hline 10 & sunnny & low & normal & no & yes \\
\hline 11 & sunnny & low & normal & yes & yes \\
\hline 12 & cloudy & low & high & yes & yes \\
\hline
\end{tabular}

Before core is calculated, discernibility matrix has to be created first. Using formulas presented in Section 2.1 for calculating discernibility matrix $D M$, this type of matrix was created for Table 1 and os presented in Table 2. Matrix is symmetrical to it's diagonal, so only lower triangle is calculated. Additionally, to limit size of the table, discernibility matrix $D M$ was calculated for only 6 first objects from dataset. The result is shown in Table 2.

According to definition-based core calculation algorithm described in Section 2.4, example of core calculation for discernibility matrix $D M$ shown in Table 2 is presented below. In the beginning, core 
$C$ is empty. Two main loops make the first iteration passing through all cells of the discernibility matrix. The condition of adding the attribute to the core is checked for each of them. The first such case occurs for the cell at the intersection of objects 1 and 3 . This cell contains only one attribute - $\{o\}$. This attribute does not exist in the core, so it is added to it, so: $C=\{o\}$. After completing the remaining algorithm steps, the final core $C=\{o, t, h, w\}$, what corresponds to $C=\{$ outlook, temp, humidity, windy $\}$.

Table 2. Discernibility matrix for 6 first objects from exemplary dataset

\begin{tabular}{ccccccc}
\hline ID & $\mathbf{1}$ & $\mathbf{2}$ & $\mathbf{3}$ & $\mathbf{4}$ & $\mathbf{5}$ & $\mathbf{6}$ \\
\hline $\mathbf{1}$ & $\emptyset$ & & & & & \\
\hline $\mathbf{2}$ & $\emptyset$ & $\emptyset$ & & & & \\
\hline $\mathbf{3}$ & $\{o\}$ & $\{o, w\}$ & $\emptyset$ & & & \\
\hline $\mathbf{4}$ & $\{t\}$ & $\{t, w\}$ & $\emptyset$ & $\emptyset$ & & \\
\hline $\mathbf{5}$ & $\{h\}$ & $\{h, w\}$ & $\emptyset$ & $\emptyset$ & $\emptyset$ & \\
\hline $\mathbf{6}$ & $\emptyset$ & $\emptyset$ & $\{o, h, w\}$ & $\{t, h, w\}$ & $\{w\}$ & $\emptyset$ \\
\hline
\end{tabular}

\subsection{Data to Conduct Experimental Re- search}

In this paper, we present the results of the conducted experiments using two datasets: Poker Hand Dataset (created by Robert Cattral and Franz Oppacher) and data about children with insulindependent diabetes mellitus (type 1) (created by Jaroslaw Stepaniuk).

First dataset was obtained from UCI Machine Learning Repository [15]. Each of 1000000 records is an example of a hand consisting of five playing cards drawn from a standard deck of 52 . Each card is described using two attributes (suit and rank), for a total of 10 predictive attributes. There is one decision attribute that describes the "Poker Hand". Decision attribute describes 10 possible combinations of cards in descending probability in the dataset: nothing in hand, one pair, two pairs, three of a kind, straight, flush, full house, four of a kind, straight flush, royal flush

Insulin-dependent diabetes mellitus is a chronic disease of the body's metabolism characterized by an inability to produce enough insulin to process carbohydrates, fat, and protein efficiently. Treatment requires injections of insulin. Twelve condition attributes, which include the results of physical and laboratory examinations and one decision attribute (microalbuminuria) describe the database used in our experiments. The data collection so far consists of 107 cases. The database is shown at the end of the paper [23]. A detailed analysis of the above data (only with the use of software systems) is in chapter 6 of the book [24].

The Poker Hand database was used for creating smaller datasets consisting of 1000 to 500000 of objects by selecting given number of rows of original dataset maintaining decision class distribution. Diabetes database was used for generating bigger datasets consisting of 1000 to 1000000 of objects. New datasets were created by multiplying the rows of original dataset.

Created datasets had to be transformed to binary version. Numerical values were discretized and each attributes' value was encoded using four bits for both datasets. Every single object was described on 44 bits for Poker Hand and 52 bits for Diabetes. To fit to memory boundaries in both cases, objects descriptions had to be extended to 64 bits words filling unused attributes with binary 0 's.

\section{Hardware Implementation}

Block diagram of the hardware implementation of the algorithm CORE-PHIDM is shown on Figure 1. System consists of the following blocks:

- $R A M_{c m n}$ - a part of the decision table that is compared with the parts of the decision table stored in the other subCORE Generator Blocks;

- subCORE Generator Block - a block (described later in this section) that performs comparison between two parts of the decision table.

With every iteration of loop 2-23 (see COREPHIDM algorithm in Section 2.3) $R A M_{c m n}$ is filled with a part of the decision table (in line 3). Every subCORE also contains the parts of the decision table. subCORE calculates the value of the sub-core in a way described in lines 7-19. Because there can be more than one instance of the subCORE, loop 7-19 can be multiplied and executed in parallel for faster calculations. 


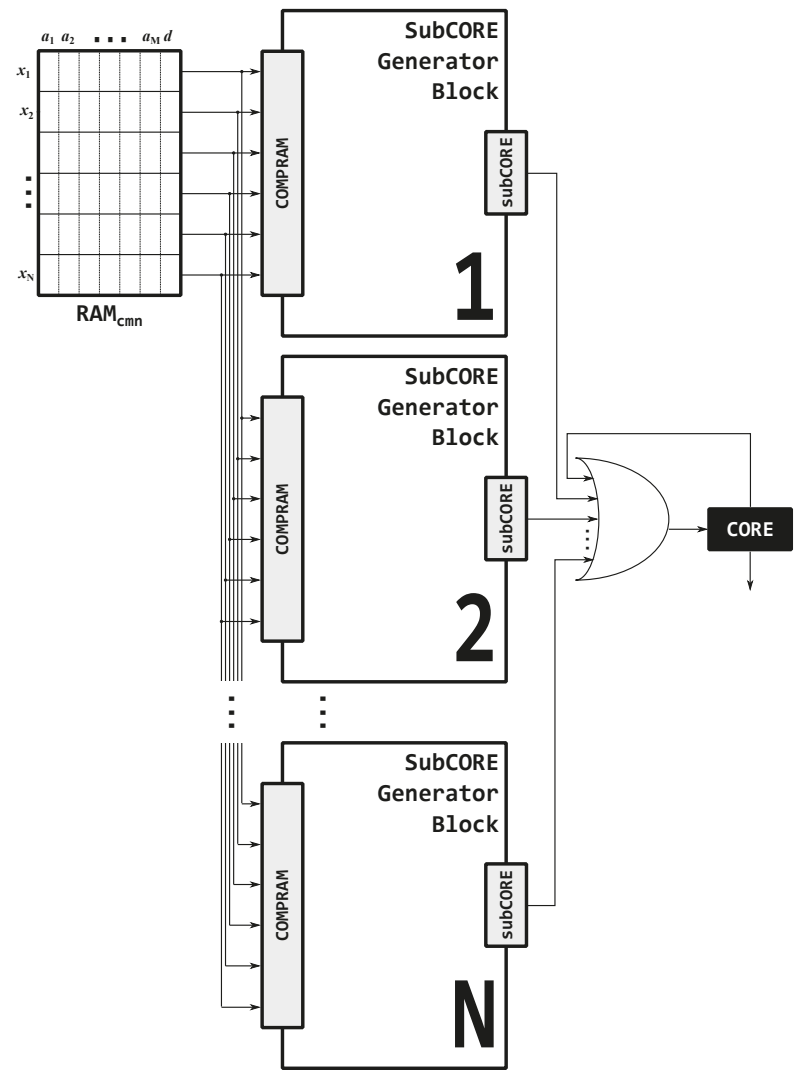

Figure 1. Main system architecture

Block diagram of the subCORE is shown in Figure 2. Block consists of the following components:

- $C B$ - comparator block, that performs comparison between two objects from decision table; when attributes have different values the corresponding bit is equal 1, otherwise 0 (line 11);

- $S D$ - singleton detector passes from input to output only singletons; when the value on the input is not singleton, the output consists of all 0's (line 16);

- OR gates cascade - every output of $S D$ is $O R^{\prime}$ ed and the result is subCORE (line 17);

- $R A M_{n}-\mathrm{RAM}$ for storing the part of the decision table (line 5);

- MUX $X_{n}$ - multiplexer controlled by Control Logic, that selects the following object for comparison (line 7);

- Control Logic - block that generates the sequence for selecting the following objects from RAM (line 7).

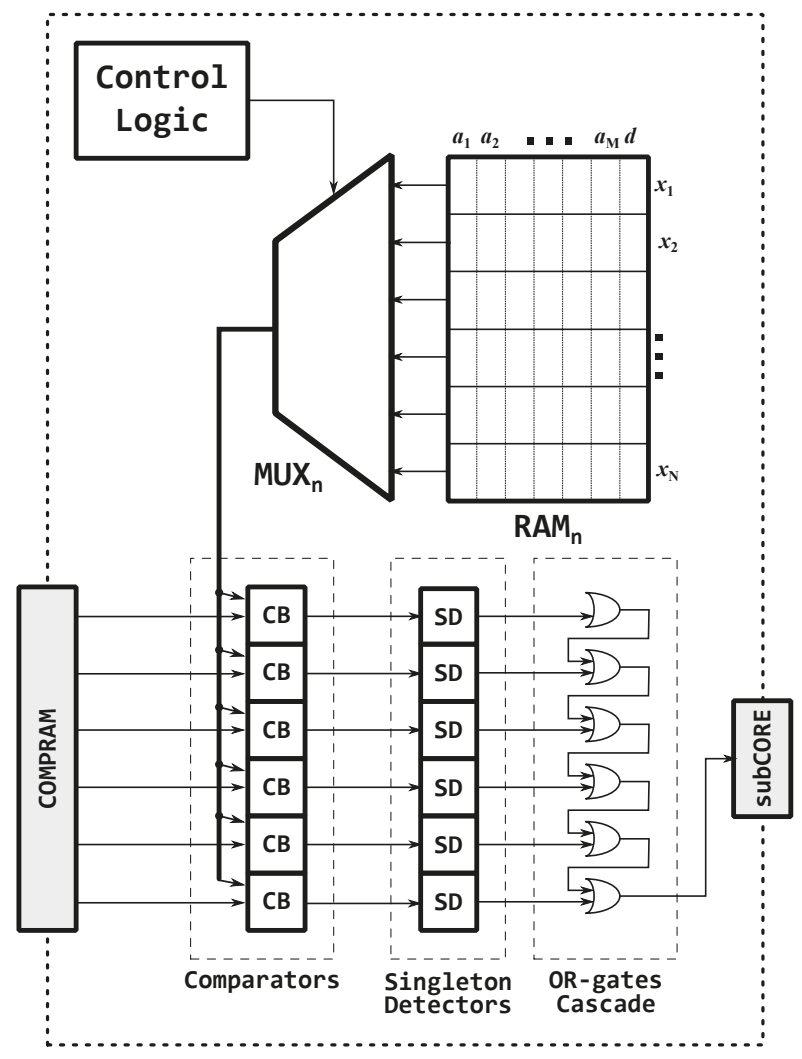

Figure 2. subCORE Generator Block block diagram

Data from $R A M_{c m n}$, consisting the objects from decision table is passed to the $C B$ 's. Second input of every $C B$ is connected to $M U X_{n}$, that selects the following object from $R A M_{n}$. All $C B$ 's outputs are filtered by the $S D$, and then $O R$ 'ed together to obtain the sub-core.

\subsection{Example of Operation}

For the clarity of presentation, only the most important data flow operations are shown. Hardware processing unit require dataset to be transformed into binary version. Dataset shown in Table 1 after transformation is presented in Table 3.

For clarity of presentation and with correspondce to CORE-PHIDM algorithm described in Section 2.3 example shows most simple configuration of CORE-PHIDM block: one instance of subCORE module with two memory blocks denoted as $R A M_{c m n}$ (common RAM connected to all modules) and $R A M_{1}$ (RAM for first SubCORE module). 
Values of the CORE-PHIDM block inputs are:

$$
\begin{aligned}
& \text { - RAM } M_{c m n}=0011101111101101010110011 \\
& 01011110100010110011100011100111100 \text {, } \\
& \text { - } R A M_{1}=0011101111101101010110011 \\
& 01011110100010110011100011100111100 \text {, }
\end{aligned}
$$

what corresponds to data in exemplary binary decision table. Most significant bit (MSB) describes decision attribute, while LSB correlates to outlook attribute.

Table 3. Exemplary binary dataset consisting of 4 condition and 1 decision attribute

\begin{tabular}{cccccc}
\hline ID & outlook & temp & humidity & windy & mixing \\
\hline 1 & 1 & 1 & 1 & 0 & 0 \\
\hline 2 & 1 & 1 & 1 & 1 & 0 \\
\hline 3 & 0 & 1 & 1 & 0 & 1 \\
\hline 4 & 1 & 0 & 1 & 0 & 1 \\
\hline 5 & 1 & 1 & 0 & 0 & 1 \\
\hline 6 & 1 & 1 & 0 & 1 & 0 \\
\hline 7 & 0 & 1 & 0 & 1 & 1 \\
\hline 8 & 1 & 0 & 1 & 0 & 0 \\
\hline 9 & 1 & 1 & 0 & 0 & 1 \\
\hline 10 & 1 & 0 & 0 & 0 & 1 \\
\hline 11 & 1 & 0 & 0 & 1 & 1 \\
\hline 12 & 0 & 0 & 1 & 1 & 1 \\
\hline
\end{tabular}

Muliplexer $M U X_{n}$ chooses $I D_{1}$ object from $R A M_{\text {cmn }}$ represented by 00111 in the first clock cycle. This object is compared with all remaining objects in $R A M_{1}$ by comparators $C B$. Calculated value corresponds to the first column of discernibility matrix created for the dataset. Binary word created by comparators is 000000000001 001001000000110100000100011011101011. Each of 4-bit subsequences is passed to given OR gate connected in cascade. Parallelly, whole word created by comparators is routed to the $S D$ block that detects 4-bits long subsequences containing only one logical 1 . Result created by $S D$ is 001110001000. Each bit of created value controls single OR gate in cascade. MSB is connected to the first OR gate. Values of inputs and outputs of every gate are:

$$
\begin{aligned}
-I N_{1 C B} & =0000 ; \quad I N_{1 P R E V}=0000 ; I N_{1 S D}=0 ; \\
O U T_{1} & =0000, \\
-I N_{2 C B} & =0000 ; \quad I N_{2 P R E V}=0000 ; I N_{2 S D}=0 ; \\
O U T_{2} & =0000,
\end{aligned}
$$

$$
\begin{aligned}
-I N_{3 C B} & =0001 ; I N_{3 P R E V}=0000 ; I N_{3 S D}=1 ; \\
O U T_{3} & =0001, \\
-I N_{4 C B} & =0010 ; I N_{4 P R E V}=0001 ; I N_{4 S D}=1 ; \\
O U T_{4} & =0011, \\
-I N_{5 C B} & =0100 ; I N_{5 P R E V}=0011 ; I N_{5 S D}=1 ; \\
O U T_{5} & =0111, \\
-I N_{6 C B} & =0000 ; I N_{6 P R E V}=0111 ; I N_{6 S D}=0 ; \\
O U T_{6} & =0111, \\
-I N_{7 C B} & =1101 ; I N_{7 P R E V}=0111 ; I N_{7 S D}=0 ; \\
O U T_{7} & =0111, \\
-I N_{8 C B} & =0000 ; I N_{8 P R E V}=0111 ; I N_{8 S D}=0 ; \\
O U T_{8} & =0111, \\
-I N_{9 C B} & =0100 ; I N_{9 P R E V}=0111 ; I N_{9 S D}=1 ; \\
O U T_{9} & =0111, \\
-I N_{10 C B} & =0110 ; I N_{10 P R E V}=0111 ; I N_{10 S D}=0 ; \\
O U T_{10} & =0111, \\
-I N_{11 C B} & =1110 ; I N_{11 P R E V}=0111 ; I N_{11 S D}=0 ; \\
O U T_{11} & =0111, \\
-I N_{12 C B} & =1011 ; I N_{12 P R E V}=0111 ; I N_{12 S D}=0 ; \\
O U T_{12} & =0111,
\end{aligned}
$$

$I N_{C B}$ input corresponds to value calculated by given comparator from $C B$ block. $I N_{P R E V}$ input is connected with $O U T$ output of preceding gate. Output of last gate is passed to TEMP register.

In next clock cycles CORE-PHIDM module processes rest of the objects in $R A M_{c m n}$ by comparing them with remaining part of dataset in $R A M_{1}$. Every time previous value of CORE output is merged with it's current value and the subCORE by OR operation.

Finally created core is $C=1111$, what corresponds to $C=\{$ outlook, temp, humidity, windy $\}$.

\section{Experimental Results}

Software version of CORE-PHIDM algorithm described in Section 2.3 was implemented in C language. It should be noted, that implementation used only one PC CPU core to create basis for comparision between PC and FPGA-based solution. The results of the software execution were obtained using a PC equipped with an 8 GB RAM and 4-core Intel Core i7 3632QM with maximum $3.2 \mathrm{GHz}$ in 
Turbo mode clock speed running Windows 10 operational system. The source code of application was compiled using the GNU GCC 9.2 compiler.

Quartus II 13.1 was used for design, compilation, synthesis and verifying simulation of the hardware implementation in VHDL language. Synthesized hardware blocks were downloaded and run on TeraSIC DE-3 equipped with Stratix III EP3SL150F1152C2N FPGA chip. FPGA clock running at $50 \mathrm{MHz}$ for the sequential parts of the project was derived from development board oscillator. Implemented CORE-PHIDM algorithm is presented in Section 2.3.

Table 4. Comparison of execution time between hardware and software implementation of CORE-PHIDM algorithm for both datasets using 1 instance of subCORE module

\begin{tabular}{ccccc}
\hline Objects & $t_{H}$ & $t_{S}$ & $C=\frac{t_{S}}{t_{H}}$ & $C_{c l k}=64 \frac{t_{S}}{t_{H}}$ \\
\hline- & {$[\underline{\mathrm{s}}]$} & {$[\mathrm{s}]$} & - & - \\
\hline \multicolumn{5}{c}{ Poker Hand dataset } \\
\hline $1 \mathrm{k}$ & 0.003 & 0.033 & 10.875 & 695.992 \\
\hline $2.5 \mathrm{k}$ & 0.013 & 0.143 & 11.119 & 711.632 \\
\hline $5 \mathrm{k}$ & 0.055 & 0.603 & 10.951 & 700.876 \\
\hline $10 \mathrm{k}$ & 0.207 & 2.410 & 11.623 & 743.851 \\
\hline $25 \mathrm{k}$ & 1.225 & 14.721 & 12.015 & 768.940 \\
\hline $50 \mathrm{k}$ & 4.710 & 58.726 & 12.469 & 798.043 \\
\hline $100 \mathrm{k}$ & 21.737 & 237.942 & 10.946 & 700.572 \\
\hline $250 \mathrm{k}$ & 130.947 & 1515.449 & 11.573 & 740.674 \\
\hline $500 \mathrm{k}$ & 506.225 & 6092.916 & 12.036 & 770.302 \\
\hline $1 \mathrm{M}$ & 1850.523 & 24313.094 & 13.138 & 840.864 \\
\hline \multicolumn{5}{c}{ Diabetes dataset } \\
\hline $1 \mathrm{k}$ & 0.003 & 0.018 & 5.911 & 378.325 \\
\hline $2.5 \mathrm{k}$ & 0.013 & 0.078 & 6.044 & 386.827 \\
\hline $5 \mathrm{k}$ & 0.055 & 0.328 & 5.953 & 380.980 \\
\hline $10 \mathrm{k}$ & 0.207 & 1.31 & 6.318 & 404.340 \\
\hline $25 \mathrm{k}$ & 1.225 & 8.002 & 6.531 & 417.978 \\
\hline $50 \mathrm{k}$ & 4.710 & 34.216 & 7.265 & 464.970 \\
\hline $100 \mathrm{k}$ & 21.737 & 135.309 & 6.225 & 398.390 \\
\hline $250 \mathrm{k}$ & 130.947 & 861.781 & 6.581 & 421.194 \\
\hline $500 \mathrm{k}$ & 506.225 & 3464.821 & 6.844 & 438.043 \\
\hline $1 \mathrm{M}$ & 1850.523 & 13825.976 & 7.471 & 478.169 \\
\hline \multicolumn{5}{c}{$\mathrm{P}$}
\end{tabular}

NIOS II softcore processor, as well as most parts of embedded system were instantiated using Qsys 13.1 tool. Software for NIOS II was implemented in C language using NIOS II Software Build Tools for Eclipse IDE.

Timing results were obtained using LeCroy waveSurfer 104MXs-B (1 GHz bandwidth, 10 GS/s) oscilloscope. For longer times, hardware time measurement units instantiated inside FPGA were used.
It should be noticed, that PCs clock is $\frac{c k_{P C}}{c k_{F P G A}}=$ 64 times faster than development boards clock source.

Table 5. Comparison of execution time between hardware and software implementation of

CORE-PHIDM algorithm for both datasets using 2 instances of SUbCORE module

\begin{tabular}{ccccc}
\hline Objects & $t_{H}$ & $t_{S}$ & $C=\frac{t_{S}}{t_{H}}$ & $C_{c l k}=64 \frac{t_{S}}{t_{H}}$ \\
\hline- & {$[\underline{s}]$} & {$[\mathrm{s}]$} & - & - \\
\hline \multicolumn{5}{c}{ Poker Hand dataset } \\
\hline $1 \mathrm{k}$ & 0.002 & 0.033 & 17.770 & 1137.250 \\
\hline $2.5 \mathrm{k}$ & 0.008 & 0.143 & 18.169 & 1162.806 \\
\hline $5 \mathrm{k}$ & 0.0340 & 0.603 & 17.894 & 1145.231 \\
\hline $10 \mathrm{k}$ & 0.127 & 2.410 & 18.991 & 1215.453 \\
\hline $25 \mathrm{k}$ & 0.750 & 14.721 & 19.632 & 1256.448 \\
\hline $50 \mathrm{k}$ & 2.882 & 58.726 & 20.375 & 1304.003 \\
\hline $100 \mathrm{k}$ & 13.303 & 237.942 & 17.886 & 1144.734 \\
\hline $250 \mathrm{k}$ & 80.139 & 1515.449 & 18.910 & 1210.261 \\
\hline $500 \mathrm{k}$ & 309.807 & 6092.916 & 19.667 & 1258.674 \\
\hline $1 \mathrm{M}$ & 1132.511 & 24313.094 & 21.468 & 1373.971 \\
\hline \multicolumn{5}{c}{ Diabetes dataset } \\
\hline $1 \mathrm{k}$ & 0.002 & 0.018 & 9.659 & 618.183 \\
\hline $2.5 \mathrm{k}$ & 0.008 & 0.078 & 9.876 & 632.075 \\
\hline $5 \mathrm{k}$ & 0.034 & 0.328 & 9.727 & 622.521 \\
\hline $10 \mathrm{k}$ & 0.127 & 1.31 & 10.323 & 660.692 \\
\hline $25 \mathrm{k}$ & 0.750 & 8.002 & 10.672 & 682.977 \\
\hline $50 \mathrm{k}$ & 2.882 & 34.216 & 11.871 & 759.761 \\
\hline $100 \mathrm{k}$ & 13.303 & 135.309 & 10.171 & 650.969 \\
\hline $250 \mathrm{k}$ & 80.139 & 861.781 & 10.754 & 688.232 \\
\hline $500 \mathrm{k}$ & 309.807 & 3464.821 & 11.184 & 715.762 \\
\hline $1 \mathrm{M}$ & 1132.511 & 13825.976 & 12.208 & 781.328 \\
\hline \multicolumn{5}{c}{} \\
\hline \multicolumn{5}{c}{}
\end{tabular}

All calculations were performed using datasets described in Section 2.5 with sizes between 1000 and 1000000 objects. Data was preprocessed on PC in terms of binary transformation and discretization for all cases.

Table 4 presents results of the time elapsed for hardware $\left(t_{H}\right)$ and software $\left(t_{S}\right)$ solution using indirect row-by-row discernibility matrix calculation (algorithm CORE-PHIDM described in Section 2.3) for both datasets.

Hardware based solution in first case uses 1 instance of subCORE generator block. Parts of dataset are stored in $R A M_{c m n}$ and $R A M_{1}$.

Last two columns in following tables describe the speed-up factor without $(C)$ and with $\left(C_{c l k}\right)$ taking clock speed factor between PC and FPGA into consideration. Abbreviations in objects number are: $k=10^{3}, M=10^{6}$. 
Table 5 shows results for time elapsed for hardware $\left(t_{H}\right)$ and software $\left(t_{S}\right)$ solution using indirect row-by-row discernibility matrix calculation for both datasets, where hardware part, in this case, uses 2 instances of SUbCORE module. Parts of dataset are stored in $R A M_{c m n}, R A M_{1}$ and $R A M_{2}$.

Table 6 presents results for time elapsed for hardware $\left(t_{H}\right)$ and software $\left(t_{S}\right)$ solution using indirect row-by-row discernibility matrix calculation for both datasets, where hardware part in this case uses 4 instances of SUbCORE module. Parts of dataset were stored in $R A M_{c m n}, R A M_{1}, R A M_{2}$, $R A M_{3}$ and $R A M_{4}$.

Table 6. Comparison of execution time between hardware and software implementation of CORE-PHIDM algorithm for both datasets using 4 instances of subCORE module

\begin{tabular}{ccccc}
\hline Objects & $t_{H}$ & $t_{S}$ & $C=\frac{t_{S}}{t_{H}}$ & $C_{c l k}=64 \frac{t_{S}}{t_{H}}$ \\
\hline- & {$[\underline{\mathrm{s}}]$} & {$[\underline{\mathrm{s}}]$} & - & - \\
\hline \multicolumn{5}{c}{ Poker Hand dataset } \\
\hline $1 \mathrm{k}$ & 0.001 & 0.033 & 30.741 & 1967.443 \\
\hline $2.5 \mathrm{k}$ & 0.005 & 0.143 & 31.432 & 2011.655 \\
\hline $5 \mathrm{k}$ & 0.019 & 0.603 & 30.957 & 1981.249 \\
\hline $10 \mathrm{k}$ & 0.073 & 2.410 & 32.855 & 2102.733 \\
\hline $25 \mathrm{k}$ & 0.433 & 14.721 & 33.963 & 2173.656 \\
\hline $50 \mathrm{k}$ & 1.666 & 58.726 & 35.249 & 2255.924 \\
\hline $100 \mathrm{k}$ & 7.690 & 237.942 & 30.944 & 1980.390 \\
\hline $250 \mathrm{k}$ & 46.323 & 1515.449 & 32.715 & 2093.752 \\
\hline $500 \mathrm{k}$ & 179.079 & 6092.916 & 34.024 & 2177.506 \\
\hline $1 \mathrm{M}$ & 654.631 & 24313.094 & 37.140 & 2376.970 \\
\hline \multicolumn{5}{c}{ Diabetes dataset } \\
\hline $1 \mathrm{k}$ & 0.001 & 0.018 & 16.710 & 1069.457 \\
\hline $2.5 \mathrm{k}$ & 0.005 & 0.078 & 17.086 & 1093.490 \\
\hline $5 \mathrm{k}$ & 0.019 & 0.328 & 16.828 & 1076.962 \\
\hline $10 \mathrm{k}$ & 0.073 & 1.31 & 17.859 & 1142.998 \\
\hline $25 \mathrm{k}$ & 0.433 & 8.002 & 18.462 & 1181.550 \\
\hline $50 \mathrm{k}$ & 1.666 & 34.216 & 20.537 & 1314.387 \\
\hline $100 \mathrm{k}$ & 7.690 & 135.309 & 17.596 & 1126.176 \\
\hline $250 \mathrm{k}$ & 46.323 & 861.781 & 18.604 & 1190.641 \\
\hline $500 \mathrm{k}$ & 179.079 & 3464.821 & 19.348 & 1238.269 \\
\hline $1 \mathrm{M}$ & 654.631 & 13825.976 & 21.120 & 1351.697 \\
\hline \multicolumn{5}{c}{}
\end{tabular}

FPGA resources utilization is fixed for given hardware core calculation configuration and is independent of the input dataset size. Datasets are divided into parts which are processed by the module. Different modules configurations for COREPHIDM use:

- 21562 Logical Elements (LE) with 1 instance of subCORE module,
- 33234 Logical Elements (LE) with 2 instances of subCORE module,

- 45668 Logical Elements (LE) with 4 instances of subCORE module.

There are 113600 Logical Elements (LE) available in Startix III FPGA. These numbers also include resources consumed by NIOS II processor.

Figure 3 presents a graphs showing the relationship between the number of objects and execution time for hardware and software solutions using different configurations of subCORE modules for CORE-PHIDM algorithm. Both axes have the logarithmic scale.

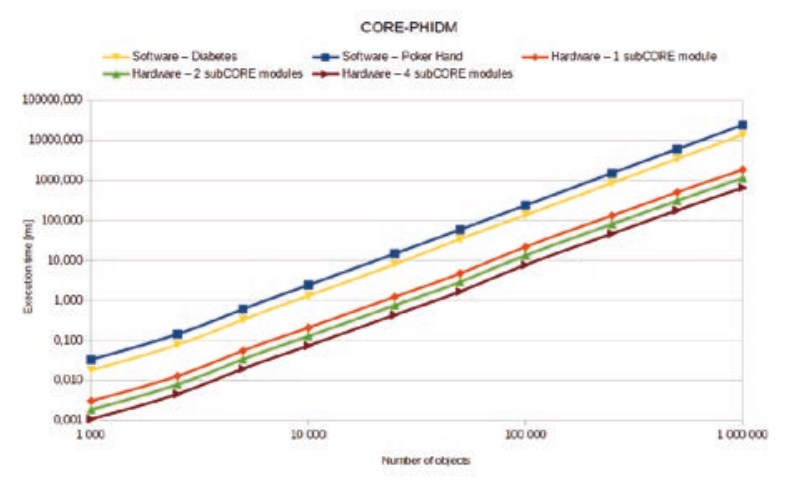

Figure 3. Relationship between number of objects and calculation time for hardware and software implementation of CORE-PHIDM for different subCORE module configurations

Presented results show big increase in the speed of data processing for all presented solutions. Hardware module execution time compared to the software implementation using row-by-row discernibility matrix calculation (algorithm CORE-PHIDM) is 5 ( 1 instance of subCORE module) to 37 (4 instances of subCORE module) times faster. If we take clock speed difference between PC and FPGA under consideration, these results are much better average speed-up factor is 378 ( 1 instance) to 2376 (4 instances). Speed-up factor is almost constant for given configuration of subCORE module and is similar for all sizes of processed datasets.

Hardware processing times for given algorithm for both datasets are the same - it doesn't matter what is the width in bits of single object from dataset, unless it fits in assumed memory boundary. Hardware processing unit takes the same time to finish the calculation for every object size, because 
it always performs the same type of operation. This is true for all configurations of presented hardware core calculation modules.

It should be noted, that average speed-up factor related to number of instances of core calculation module for CORE-PHIDM is not linear and is equal to:

- 1.634 for configuration of 2 instances of core calculation module,

- 2.827 for configuration of 4 instances of core calculation module.

Decreasing speed-up factor is realted to NIOS II processor overhead needed for binary data copying to $R A M_{n}$ memories what needs increasing time for this operation.

Let comparison of attribute value between two objects or retrieving the element from discernibility matrix be an elementary operation. $k$ denotes number of conditional attributes, $n$ is the number of objects in decision table and $p$ is number of subCORE module instances. Computational complexity of software implementation for the core calculation using CORE-PHIDM algorithm is $\Theta\left(k n^{2}\right)$. Using hardware implementation, complexity of core calculation is $\Theta\left(\frac{n^{2}}{p}\right)$. The $k$ is missing in hardware implementation, because presented solutions perform comparison between all attributes in $\Theta(1)$ - all attributes values between two objects are compared in single clock cycle. Additionally, core module performs comparisons between many objects at time. In most cases $k<<n$, while $p<<k$, so we can say, that computational complexity for software and hardware implementations are the same.

\section{Conclusions and Future Re- search}

Hardware parallel implementation of a core calculation algorithm gives us a big acceleration in comparison to a software solution. This approach is the main direction of using scalable decision systems in solutions demanding results of calculations in a short time.

Core hardware calculation units were not optimized for performance. Processing time can be substantially reduced by increasing FPGA clock fre- quency and by introducing triggering on both clock edges. The hardware solution presented in this paper is easily scalable. Multiplying core calculation blocks improved the processing speed. Currently, 4 parallel units occupy only about $50 \%$ of a pretty small FPGA.

Further research will focus on checking different sizes of modules, optimizing the modules for performance as well as optimizing the data transfer between the decision table and core calculation units.

The type of processed data must also be taken into consideration. The presented solution is suitable for consistent datasets. This approach doesn't handle databases with missing values properly. In recent years increasing the popularity of systems dealing with incomplete information is gaining popularity. The algorithm and its implementation should be modified for processing this type of data. Examples of such an approach for software solutions can be found in e.g. [26].

\section{Acknowledgments}

The work was supported by the grant W/WI/2/2020, WZ/WI-IIT/2/2020 from Bialystok University of Technology and funded with resources for research by the Ministry of Science and Higher Education in Poland.

\section{References}

[1] Borowik, G.; Jankowski, J.; Kowalski K. Fast algorithm for feature extraction. Proceedings of SPIE 9662, In Photonics Applications in Astronomy, Communications, Industry, and High-Energy Physics Experiments, Proceedings of SPIE 2015, pp. 1110-1117.

[2] Choromański, M.; Grześ, T.; Hońko, P. Two FPGA Devices in the Problem of Finding Minimal Reducts. In Lecture Notes in Computer Science, Publisher: Springer, 2019, Vol. 11703, pp. 410-420.

[3] Czołombitko, M.; Stepaniuk, J. Generating core based on discernibility measure and MapReduce. Proceedings of the Pattern recognition and machine intelligence: 6th International conference, PReMI 2015, Warsaw, Poland, June 30-July 3, 2015, Lecture Notes in Computer Science, vol. 9124, pp. 367-376. 
[4] T. Geng et al., O3BNN-R: An Out-of-Order Architecture for High-Performance and Regularized BNN Inference, in IEEE Transactions on Parallel and Distributed Systems, vol. 32, no. 1, 1 Jan. 2021, doi: 10.1109/TPDS.2020.3013637, pp. 199213.

[5] Grześ T., Kopczyński M. Hardware Implementation on Field Programmable Gate Array of TwoStage Algorithm for Rough Set Reduct Generation. In Lecture Notes in Computer Science, Publisher: Springer, 2019, Vol. 11499, pp. 495-506.

[6] Y. Liang, L. Lu and J. Xie, OMNI: A Framework for Integrating Hardware and Software Optimizations for Sparse CNNs in IEEE Transactions on Computer-Aided Design of Integrated Circuits and Systems, doi: 10.1109/TCAD.2020.3023903.

[7] Z. -N. Li, C. Zhu, Y. -L. Gao, Z. -K. Wang and J. Wang, AlphaGo Policy Network: A DCNN Accelerator on FPGA in IEEE Access, doi: 10.1109/ACCESS.2020.3023739.

[8] Kanasugi, A.; Yokoyama, A. A basic design for rough set processor. In Proceedings of the 15th Annual Conference of Japanese Society for Artificial Intelligence, 2001.

[9] Kopczyński, M.; Stepaniuk, J. Hardware Implementations of Rough Set Methods in Programmable Logic Devices. In Rough Sets and Intelligent Systems - Professor Zdzisław Pawlak in Memoriam, Intelligent Systems Reference Library 43, Heidelberg, Springer; 2013, pp. 309-321.

[10] Kopczyński, M.; Grześ, T.; Stepaniuk, J. FPGA in Rough-Granular Computing: Reduct Generation. In proceedings of the 2014 IEEE/WCI/ACM International Joint Conferences on Web Intelligence Vol.2, Warsaw, IEEE Computer Society, 2014, pp. 364-370.

[11] Kopczyński, M.; Grześ, T.; Stepaniuk, J. Generating core in rough set theory: Design and implementation on FPGA. In Lecture Notes in Computer Science Vol. 8537, Berlin, Springer-Verlag, 2014, pp. 209-216.

[12] Kopczyński, M.; Grześ, T.; Stepaniuk, J. Computation of Cores in Big Datasets: An FPGA Approach. In Lecture Notes in Computer Science Vol.9436, Berlin, Springer-Verlag, 2015, pp. 153-163.

[13] Kopczyński, M.; Grześ, T.; Stepaniuk, J. Core for Large Datasets: Rough Sets on FPGA. Fundamenta Informaticae 2016, 147, pp. 241-259.

[14] Lewis, T.; Perkowski, M.; Jozwiak, L. Learning in Hardware: Architecture and Implementation of an FPGA-Based Rough Set Machine. In proceedings of the Euromicro, 25th Euromicro Conference (EUROMICRO '99), Volume 1; 1999, pp. 13-26.
[15] Lichman, M. UCI Machine Learning Repository [http://archive.ics.uci.edu/ml]. Irvine, CA: University of California, School of Information and Computer Science; 2013.

[16] Muraszkiewicz, M.; Rybiński, H. Towards a Parallel Rough Sets Computer In: Rough Sets, Fuzzy Sets and Knowledge Discovery. Springer-Verlag; 1994, pp. 434-443.

[17] Marz, N.; Warren, J.H. Big Data: Principles and best practices of scalable realtime data systems. Manning Publications Co. Greenwich; 2015.

[18] N. Narsale and V. Agarwal, Implementation Of LEM2 algorithm On FPGA, 2019 3rd International Conference on Electronics, Communication and Aerospace Technology (ICECA), Coimbatore, India, 2019, doi: 10.1109/ICECA.2019.8822143, pp. $1-5$.

[19] Nguyen, S. H.; Nguyen H. S. Some Efficient Algorithms for Rough Set Methods. In Sixth International Conference on Information Processing and Management of Uncertainty on Knowledge Based Systems IPMU'1996, volume III, Granada, Spain, July 1-5 1996, pp 1451-1456.

[20] Nguyen, H.S. Approximate Boolean Reasoning: Foundations and Applications in Data Mining. Transactions on Rough Sets V, Lecture Notes in Computer Science Vol. 4100, Berlin, SpringerVerlag; 2006, pp. 334-506.

[21] Pawlak, Z. Elementary rough set granules: Toward a rough set processor. In Rough-Neurocomputing: Techniques for Computing with Words, Cognitive Technologies, Springer-Verlag, Berlin, Germany; 2004, p. 5-14.

[22] Pawlak, Z.; Skowron, A. Rudiments of rough sets. Information Sciences; 2007; 177(1), p. 3-27.

[23] Stepaniuk, J. Knowledge discovery by application of rough set models. In Rough Set Methods and Applications. New Developments in Knowledge Discovery, Information Systems, Physica-Verlag, Heidelberg; 2000, p. 137-233.

[24] Stepaniuk, J. Rough-Granular Computing in Knowledge Discovery and Data Mining. Springer, 2008.

[25] Stepaniuk, J.; Kopczyński, M.; Grześ, T. The First Step Toward Processor for Rough Set Methods. Fundamenta Informaticae 2013; 127, pp. 429-443.

[26] Sun, L.; Xu, J.; Li, Y. A Feature Selection Approach of Inconsistent Decision Systems in Rough Set. Journal of Computers, vol. 9, Academy Publisher; 2014, pp. 1333-1340. 
[27] Tiwari, K.S.; Kothari, A.G. Design and Implementation of Rough Set Algorithms on FPGA: A Survey. IJARAI, 2014, 3, no. 9, pp. 14-23.

[28] Zhang, J.; Wong, J.; Pan, Y.; Li, T. A paral- lel matrix-based method for computing approximations in incomplete information systems. In IEEE Transactions on Knowledge Data Engineering, 2015, 27, p. 326-339.

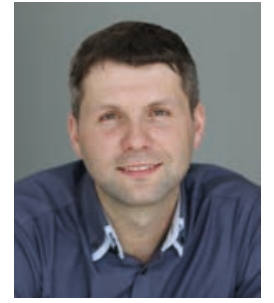

Maciej Kopczyński is an experienced professional working at the Bialystok University of Technology since 2008. He had finished his Ph.D. in 2017 mainly focusing on hardware implementation of selected AI algorithms in FPGA structures. Maciej conducts classes related to entrepreneurship, programming, web application development, artificial intelligence and algorithms. Maciej is a co-founder of four companies: Photon Entertainment, RiftCat, Anima and MakeIT. He also has extensive experience in working with industry in the aspect of implementing projects in the field of very advanced electronics (mainly RF systems) and complex software solutions.

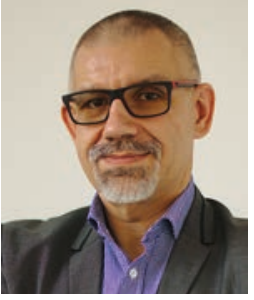

Tomasz Grześ is an experienced professional working at the Bialystok University of Technology since 1998. He had finished his Ph.D. in 2010 focusing on power minimization in sequential circuits on programmable logic devices. Tomasz conducts classes related to digital systems design, programming, computer networks, as well as embedded and mobile systems. He also has extensive experience in working with the industry in the aspect of implementing projects in the field of advanced control and management systems, firmware development, and specialized digital systems design. 